\title{
NONLOCAL DIFFUSION PROBLEMS THAT APPROXIMATE THE HEAT EQUATION WITH DIRICHLET BOUNDARY CONDITIONS
}

\author{
CARMEN CORTAZAR, MANUEL ELGUETA, AND JULIO D. ROSSI
}

\begin{abstract}
We present a model for nonlocal diffusion with Dirichlet boundary conditions in a bounded smooth domain. We prove that solutions of properly re-scaled non local problems approximate uniformly the solution of the corresponding Dirichlet problem for the classical heat equation.
\end{abstract}

\section{INTRODUCTION}

Let $J: \mathbb{R}^{N} \rightarrow \mathbb{R}$ be a nonnegative, radial, continuous function with $\int_{\mathbb{R}^{N}} J(z) d z=1$. Assume also that $J$ is strictly positive in $B(0, d)$ and vanishes in $\mathbb{R}^{N} \backslash B(0, d)$. Nonlocal evolution equations of the form

$$
u_{t}(x, t)=(J * u-u)(x, t)=\int_{\mathbb{R}^{N}} J(x-y) u(y, t) d y-u(x, t),
$$

and variations of it, have been widely used to model diffusion processes. As stated in [11] equation (1.1) models a, continuous in time, "random walk" where the probability distribution of jumping from location $y$ to location $x$ is given by $J(x-y)$. For recent references on nonlocal diffusion see, [1], [2], [3], [4], [5], [6], [10], [11], [13], [14] and references therein.

In this article we propose the following nonlocal "Dirichlet" boundary value problem: Given $g(x, t)$ defined for $x \in \mathbb{R}^{N} \backslash \Omega$ and $u_{0}(x)$ defined for $x \in \Omega$, find $u(x, t)$ such that

$$
\begin{cases}u_{t}(x, t)=\int_{\mathbb{R}^{N}} J(x-y)(u(y, t)-u(x, t)) d y, & x \in \Omega, t>0, \\ u(x, t)=g(x, t), & x \notin \Omega, t>0, \\ u(x, 0)=u_{0}(x), & x \in \Omega .\end{cases}
$$

In this model we prescribe the values of $u$ outside $\Omega$ which is the analogous of prescribing the so called Dirichlet boundary conditions for the classical heat equation. However, the boundary data is not understood in the usual sense as we will see in Remark 2.1 below. As explained before in this model the right hand side models the diffusion, the integral $\int J(x-y)(u(y, t)-$

Key words and phrases. Nonlocal diffusion, boundary value problems.

2000 Mathematics Subject Classification 35K57, 35B40. 
$u(x, t)) d y$ takes into account the individuals arriving or leaving position $x \in \Omega$ from or to other places while we are prescribing the values of $u$ outside the domain $\Omega$ by imposing $u=g$ for $x \notin \Omega$. When $g=0$ we get that any individuals that leave $\Omega$ die, this is the case when $\Omega$ is surrounded by a hostile enviroment.

Existence and uniqueness of solutions of (1.2) is proved by a fixed point argument in Section 2 where a comparison principle is also obtained.

Let us consider the classical Dirichlet problem for the heat equation,

$$
\begin{cases}v_{t}(x, t)-\Delta v(x, t)=0, & x \in \Omega, t>0, \\ v(x, t)=g(x, t), & x \in \partial \Omega, t>0, \\ v(x, 0)=u_{0}(x), & x \in \Omega .\end{cases}
$$

The nonlocal Dirichlet model (1.2) and the classical Dirichlet problem (1.3) share many properties, among them the asymptotic behavior of their solutions as $t \rightarrow \infty$ is similar as was proved in [7].

The main goal of this article is to show that the Dirichlet problem for the heat equation (1.3) can be approximated by suitable nonlocal problems of the form of (1.2).

More precisely, for a given $J$ and a given $\varepsilon>0$ we consider the rescaled kernel

$$
J_{\varepsilon}(\xi)=C_{1} \frac{1}{\varepsilon^{N}} J\left(\frac{\xi}{\varepsilon}\right), \quad \text { with } \quad C_{1}^{-1}=\frac{1}{2} \int_{B(0, d)} J(z) z_{N}^{2} d z .
$$

Here $C_{1}$ is a normalizing constant in order to obtain the Laplacian in the limit instead of a multiple of it. Let $u^{\varepsilon}(x, t)$ be the solution of

$$
\begin{cases}u_{t}^{\varepsilon}(x, t)=\int_{\Omega} \frac{J_{\varepsilon}(x-y)}{\varepsilon^{2}}\left(u^{\varepsilon}(y, t)-u^{\varepsilon}(x, t)\right) d y, & x \in \Omega, t>0, \\ u(x, t)=g(x, t), & x \notin \Omega, t>0, \\ u(x, 0)=u_{0}(x), & x \in \Omega .\end{cases}
$$

Our main result now reads as follows.

Theorem 1.1. Let $\Omega$ be a bounded $C^{2+\alpha}$ domain for some $0<\alpha<1$. Let $v \in C^{2+\alpha, 1+\alpha / 2}(\bar{\Omega} \times[0, T])$ be the solution to (1.3) and let $u^{\varepsilon}$ be the solution to (1.5) with $J_{\varepsilon}$ as above. Then, there exists $C=C(T)$ such that

$$
\sup _{t \in[0, T]}\left\|v-u^{\varepsilon}\right\|_{L^{\infty}(\Omega)} \leq C \varepsilon^{\alpha} \rightarrow 0, \quad \text { as } \varepsilon \rightarrow 0 .
$$

Related results for the Neumann problem where recently obtained in [9].

Note that the assumed regularity of $v$ is a consequence of regularity assumptions on the boundary data $g$, the domain $\Omega$ and the initial condition $u_{0}$, see [12]. 
The rest of the paper is organized as follows: in Section 2 we prove existence, uniqueness and a comparison principle for our nonlocal equation and in Section 3 we prove the convergence result.

\section{EXISTENCE, UNIQUENESS AND A COMPARISON PRINCIPLE}

Existence and uniqueness of solutions is a consequence of Banach's fixed point theorem. We look for $u \in C\left([0, \infty) ; L^{1}(\Omega)\right)$ satisfying (1.2). Fix $t_{0}>0$ and consider the Banach space $X_{t_{0}}=\left\{w \in C\left(\left[0, t_{0}\right] ; L^{1}(\Omega)\right)\right\}$ with the norm

$$
\||| w \mid\|=\max _{0 \leq t \leq t_{0}}\|w(\cdot, t)\|_{L^{1}(\Omega)} .
$$

We will obtain the solution as a fixed point of the operator $\mathcal{T}: X_{t_{0}} \rightarrow X_{t_{0}}$ defined by

$$
\mathcal{T}_{w_{0}}(w)(x, t)=w_{0}(x)+\int_{0}^{t} \int_{\mathbb{R}^{N}} J(x-y)(w(y, s)-w(x, s)) d y d s,
$$

where we impose

$$
w(x, t)=g(x, t), \quad \text { for } x \notin \Omega .
$$

Lemma 2.1. Let $w_{0}, z_{0} \in L^{1}(\Omega)$, then there exists a constant $C$ depending on $J$ and $\Omega$ such that

$$
\left\|\left|\mathcal{T}_{w_{0}}(w)-\mathcal{T}_{z_{0}}(z)\right||| \leq C t_{0}|||w-z|||+\right\| w_{0}-z_{0} \|_{L^{1}(\Omega)}
$$

for all $w, z \in X_{t_{0}}$.

Proof. We have

$$
\begin{array}{r}
\int_{\Omega}\left|\mathcal{T}_{w_{0}}(w)(x, t)-\mathcal{T}_{z_{0}}(z)(x, t)\right| d x \leq \int_{\Omega}\left|w_{0}-z_{0}\right|(x) d x \\
+\int_{\Omega} \mid \int_{0}^{t} \int_{\mathbb{R}^{N}} J(x-y)[(w(y, s)-z(y, s)) \\
-(w(x, s)-z(x, s))] d y d s \mid d x .
\end{array}
$$

Hence, taking into account that $w$ and $z$ vanish outside $\Omega$,

$$
\left\|\left|\mathcal{T}_{w_{0}}(w)-\mathcal{T}_{z_{0}}(z)\left\||| \leq|| w_{0}-z_{0}\right\|_{L^{1}(\Omega)}+C t_{0}\right||| w-z \mid\right\|,
$$

as we wanted to prove.

Theorem 2.1. For every $u_{0} \in L^{1}(\Omega)$ there exists a unique solution $u$, such that $u \in C\left([0, \infty) ; L^{1}(\Omega)\right)$.

Proof. We check first that $\mathcal{T}_{u_{0}}$ maps $X_{t_{0}}$ into $X_{t_{0}}$. Taking $z_{0} \equiv 0$ and $z \equiv 0$ in Lemma 2.1 we get that $\mathcal{T}_{u_{0}}(w) \in C\left(\left[0, t_{0}\right] ; L^{1}(\Omega)\right)$ for any $w \in X_{t_{0}}$.

Choose $t_{0}$ such that $C t_{0}<1$. Now taking $z_{0} \equiv w_{0} \equiv u_{0}$ in Lemma 2.1 we get that $\mathcal{T}_{u_{0}}$ is a strict contraction in $X_{t_{0}}$ and the existence and uniqueness part of the theorem follows from Banach's fixed point theorem in the interval $\left[0, t_{0}\right]$. To extend the solution to $[0, \infty)$ we may take as initial 
data $u\left(x, t_{0}\right) \in L^{1}(\Omega)$ and obtain a solution up to $\left[0,2 t_{0}\right]$. Iterating this procedure we get a solution defined in $[0, \infty)$.

Remark 2.1. Note that in general a solution $u$ with $u_{0}>0$ and $g=0$ is strictly positive in $\bar{\Omega}$ (with a positive continuous extension to $\bar{\Omega}$ ) and vanishes outside $\bar{\Omega}$. Therefore a discontinuity occurs on $\partial \Omega$ and the boundary value is not taken in the usual "classical" sense, see [7].

We now define what we understand by sub and supersolutions.

Definition 2.1. A function $u \in C\left([0, T) ; L^{1}((\Omega))\right.$ is a supersolution of $(1.2)$ if

$$
\begin{cases}u_{t}(x, t) \geq \int_{\mathbb{R}^{N}} J(x-y)(u(y, t)-u(x, t)) d y, & x \in \Omega, t>0, \\ u(x, t) \geq g(x, t), & x \notin \Omega, t>0, \\ u(x, 0) \geq u_{0}(x), & x \in \Omega .\end{cases}
$$

As usual, subsolutions are defined analogously by reversing the inequalities.

Lemma 2.2. Let $u_{0} \in C(\bar{\Omega}), u_{0} \geq 0$, and $u \in C(\bar{\Omega} \times[0, T])$ a supersolution to (1.2) with $g \geq 0$. Then, $u \geq 0$.

Proof. Assume for contradiction that $u(x, t)$ is negative somewhere. Let $v(x, t)=u(x, t)+\varepsilon t$ with $\varepsilon$ so small such that $v$ is still negative somewhere. Then, if $\left(x_{0}, t_{0}\right)$ is a point where $v$ attains its negative minimum, there holds that $t_{0}>0$ and

$$
\begin{gathered}
v_{t}\left(x_{0}, t_{0}\right)=u_{t}\left(x_{0}, t_{0}\right)+\varepsilon>\int_{\mathbb{R}^{N}} J(x-y)\left(u\left(y, t_{0}\right)-u\left(x_{0}, t_{0}\right)\right) d y \\
=\int_{\mathbb{R}^{N}} J(x-y)\left(v\left(y, t_{0}\right)-v\left(x_{0}, t_{0}\right)\right) d y \geq 0
\end{gathered}
$$

which is a contradiction. Thus, $u \geq 0$.

Corollary 2.1. Let $J \in L^{\infty}\left(\mathbb{R}^{N}\right)$. Let $u_{0}$ and $v_{0}$ in $L^{1}(\Omega)$ with $u_{0} \geq v_{0}$ and $g, h \in L^{\infty}\left((0, T) ; L^{1}\left(\mathbb{R}^{N} \backslash \Omega\right)\right)$ with $g \geq h$. Let $u$ be a solution of $(1.2)$ with $u(x, 0)=u_{0}$ and Dirichlet datum $g$ and $v$ be a solution of (1.2) with $v(x, 0)=v_{0}$ and datum $h$. Then, $u \geq v$ a.e.

Proof. Let $w=u-v$. Then, $w$ is a supersolution with initial datum $u_{0}-v_{0} \geq$ 0 and datum $g-h \geq 0$. Using the continuity of solutions with respect to the data and the fact that $J \in L^{\infty}\left(\mathbb{R}^{N}\right)$, we may assume that $u, v \in$ $C(\bar{\Omega} \times[0, T])$. By Lemma 2.2 we obtain that $w=u-v \geq 0$. So the corollary is proved.

Corollary 2.2. Let $u \in C(\bar{\Omega} \times[0, T]$ ) (resp. v) be a supersolution (resp. subsolution) of (1.2). Then, $u \geq v$.

Proof. It follows the lines of the proof of the previous corollary. 


\section{Convergence to the heat equation}

In order to prove Theorem 1.1 let $\tilde{v}$ be a $C^{2+\alpha, 1+\alpha / 2}$ extension of $v$ to $\mathbb{R}^{N} \times[0, T]$.

Let us define the operator

$$
\tilde{L}_{\varepsilon}(z)=\frac{1}{\varepsilon^{2}} \int_{\mathbb{R}^{N}} J_{\varepsilon}(x-y)(z(y, t)-z(x, t)) d y .
$$

Then $\tilde{v}$ verifies

$$
\begin{cases}\tilde{v}_{t}(x, t)=\tilde{L}_{\varepsilon}(\tilde{v})(x, t)+F_{\varepsilon}(x, t) & x \in \Omega,(0, T], \\ \tilde{v}(x, t)=g(x, t)+G(x, t), & x \notin \Omega,(0, T], \\ \tilde{v}(x, 0)=u_{0}(x), & x \in \Omega .\end{cases}
$$

where, since $\Delta v=\Delta \tilde{v}$ in $\Omega$,

$$
F_{\varepsilon}(x, t)=-\tilde{L}_{\varepsilon}(\tilde{v})(x, t)+\Delta \tilde{v}(x, t) .
$$

Moreover as $G$ is smooth and $G(x, t)=0$ if $x \in \partial \Omega$ we have

$$
G(x, t)=O(\varepsilon), \quad \text { for } x \text { such that } \operatorname{dist}(x, \partial \Omega) \leq \varepsilon d .
$$

We set $w^{\varepsilon}=\tilde{v}-u^{\varepsilon}$ and we note that

$$
\left\{\begin{array}{llrl}
w_{t}^{\varepsilon}(x, t) & =\tilde{L}_{\varepsilon}\left(w^{\varepsilon}\right)(x, t)+F_{\varepsilon}(x, t) & & x \in \Omega,(0, T], \\
w^{\varepsilon}(x, t) & =G(x, t), & & x \notin \Omega,(0, T], \\
w^{\varepsilon}(x, 0) & =0, & & x \in \Omega .
\end{array}\right.
$$

First, we claim that, by the choice of $C_{1}$, the fact that $J$ is radially symmetric and $\tilde{u} \in C^{2+\alpha, 1+\alpha / 2}\left(\mathbb{R}^{N} \times[0, T]\right)$, we have that

$$
\sup _{t \in[0, T]}\left\|F_{\varepsilon}\right\|_{L^{\infty}(\Omega)}=\sup _{t \in[0, T]}\left\|\Delta \tilde{v}-\tilde{L}_{\varepsilon}(\tilde{v})\right\|_{L^{\infty}(\Omega)}=O\left(\varepsilon^{\alpha}\right) .
$$

In fact,

$$
\Delta \tilde{v}(x, t)-\frac{C_{1}}{\varepsilon^{N+2}} \int_{\mathbb{R}^{N}} J\left(\frac{x-y}{\varepsilon}\right)(\tilde{v}(y, t)-\tilde{v}(x, t)) d y
$$

becomes, under the change variables $z=(x-y) / \varepsilon$,

$$
\Delta \tilde{v}(x, t)-\frac{C_{1}}{\varepsilon^{2}} \int_{\mathbb{R}^{N}} J(z)(\tilde{v}(x-\varepsilon z, t)-\tilde{v}(x, t)) d z
$$

and hence (3.3) follows by a simple Taylor expansion. This proves the claim.

We proceed now to prove Theorem 1.1.

Proof of Theorem 1.1. In order to prove the theorem by a comparisonwe first look for a supersolution. Let $\bar{w}$ be given by

$$
\bar{w}(x, t)=K_{1} \varepsilon^{\alpha} t+K_{2} \varepsilon .
$$


For $x \in \Omega$ we have, if $K_{1}$ is large,

$$
\bar{w}_{t}(x, t)-\tilde{L}(\bar{w})(x, t)=K_{1} \varepsilon^{\alpha} \geq F_{\varepsilon}(x, t)=w_{t}^{\varepsilon}(x, t)-\tilde{L}_{\varepsilon}\left(w^{\varepsilon}\right)(x, t) .
$$

Since

$$
G_{\varepsilon}(x, t)=O(\varepsilon) \quad \text { for } x \text { such that } \operatorname{dist}(x, \partial \Omega) \leq \varepsilon
$$

choosing $K_{2}$ large, we obtain

$$
\bar{w}(x, t) \geq w^{\varepsilon}(x, t)
$$

for $x \notin \Omega$ such that $\operatorname{dist}(x, \partial \Omega) \leq \varepsilon d$ and $t \in[0, T]$. Moreover it is clear that

$$
\bar{w}(x, 0)=K_{2} \varepsilon>w^{\varepsilon}(x, 0)=0 .
$$

Thanks to (3.5), (3.6) and (3.7) we can apply the comparison result and conclude that

$$
w^{\varepsilon}(x, t) \leq \bar{w}(x, t)=K_{1} \varepsilon^{\alpha} t+K_{2} \varepsilon .
$$

In a similar fashion we prove that $\underline{w}(x, t)=-K_{1} \varepsilon^{\alpha} t-K_{2} \varepsilon$ is a subsolution and hence

$$
w^{\varepsilon}(x, t) \geq \underline{w}(x, t)=-K_{1} \varepsilon^{\alpha} t-K_{2} \varepsilon .
$$

Therefore

$$
\sup _{t \in[0, T]}\left\|u-u^{\varepsilon}\right\|_{L^{\infty}(\Omega)} \leq C(T) \varepsilon^{\alpha},
$$

as we wanted to prove.

Acknowledgements. Supported by Universidad de Buenos Aires under grant X066, by ANPCyT PICT No. 03-13719, by CONICET (Argentina) and by FONDECYT Project 1070944 and Coop. Int. 7050118 (Chile).

\section{REFERENCES}

[1] P. Bates and A. Chmaj. An integrodifferential model for phase transitions: stationary solutions in higher dimensions. J. Statistical Phys., 95, 1119-1139, (1999).

[2] P. Bates and A. Chmaj. A discrete convolution model for phase transitions. Arch. Rat. Mech. Anal., 150, 281-305, (1999).

[3] P. Bates, P. Fife, X. Ren and X. Wang. Travelling waves in a convolution model for phase transitions. Arch. Rat. Mech. Anal., 138, 105-136, (1997).

[4] P. Bates and J. Han. The Dirichlet boundary problem for a nonlocal Cahn-Hilliard equation. J. Math. Anal. Appl. J. Math. Anal. Appl. 311(1), 289-312, (2005).

[5] P. Bates and J. Han. The Neumann boundary problem for a nonlocal CahnHilliard equation. J. Differential Equations, 212, 235-277, (2005).

[6] C. Carrillo and P. Fife. Spatial effects in discrete generation population models. J. Math. Biol. 50(2), 161-188, (2005).

[7] E. Chasseigne, M. Chaves and J. D. Rossi. Asymptotic behavior for nonlocal diffusion equations. J. Math. Pures et Appl. 86, 271-291, (2006).

[8] C. Cortazar, M. Elgueta and J. D. Rossi. A non-local diffusion equation whose solutions develop a free boundary. Ann. Henri Poincare, 6(2), 269-281, (2005). 
[9] C. Cortazar, M. Elgueta, J. D. Rossi and N. Wolanski. How to approximate the heat equation with Neumann boundary conditions by nonlocal diffusion problems. To appear in Arch. Rat. Mech. Anal.

[10] X Chen. Existence, uniqueness and asymptotic stability of travelling waves in nonlocal evolution equations. Adv. Differential Equations, 2, 125-160, (1997).

[11] P. Fife. Some nonclassical trends in parabolic and parabolic-like evolutions. Trends in nonlinear analysis, 153-191, Springer, Berlin, 2003.

[12] A. Friedman. "Partial Differential Equations of Parabolic Type". Prentice-Hall, Englewood Cliffs, NJ, 1964.

[13] X. Wang. Metaestability and stability of patterns in a convolution model for phase transitions. J. Differential Equations, 183, 434-461, (2002).

[14] L. Zhang. Existence, uniqueness and exponential stability of traveling wave solutions of some integral differential equations arising from neuronal networks. J. Differential Equations 197(1), 162-196, (2004).

Carmen Cortazar and Manuel Elgueta

Departamento de Matemática, Universidad Catolica de Chile,

Casilla 306, Correo 22, Santiago, Chile.

E-mail address: ccortaza@mat.puc.cl, melgueta@mat.puc.cl.

Julio D. Rossi

Departamento de Matemática, FCEyN

UBA (1428) Buenos Aires, Argentina.

E-mail address: jrossi@dm.uba.ar 